\title{
Is there an association between anxiety/ depression and temporomandibular disorders in college students?
}

\author{
Letícia Bojikian CALIXTRE¹, Bruno Leonardo da Silva GRÜNINGER', Thais Cristina CHAVES ${ }^{2}$, Ana Beatriz de \\ OLIVEIRA $^{1}$
}

1- Department of Physical Therapy, Federal University of São Carlos (UFSCar), São Carlos, SP, Brazil.
2- Department of Neurosciences and Behavioral Sciences, University of São Paulo (FMRP/USP), Ribeirão Preto, SP, Brazil.

Corresponding address: Letícia Bojikian Calixtre - Universidade Federal de São Carlos, Departamento de Fisioterapia - Via Washington Luis, Km 235 - CP 676 - 13565-905 - São Carlos - SP - Brazil - e-mail: lecalixtre@hotmail.com - Phone: +55 1633519793 - Fax: +55 1633612081

Submitted: January 28, 2013 - Modification: September 5, 2013 - Accepted: October 7, 2013

\section{ABSTRACT}

\begin{abstract}
bjective: Considering the high incidence of Temporomandibular Disorders (TMD) in the population aged 15-30 years and the fact that students are exposed to stressful psychosocial factors, the purposes of this study were: to verify clinical symptoms and jaw functionality in college students with TMD according to the anxiety/depression (A/D) level and to evaluate the correlation between $A / D$ and functionality, maximum mouth opening (MMO) and pain and muscle activity. Material and Methods: Nineteen students with TMD diagnosed according to the Research Diagnostic Criteria for Temporomandibular Disorders underwent two assessments during an academic semester. The evaluations were based on questionnaires (MFIQ - Mandibular Function Impairment Questionnaire; HADS - Hospital Anxiety and Depression Scale), clinical measurements (MMO without pain, MMO and assisted MMO; palpation of joint and masticatory muscles), and electromyography. The HADS scores obtained in the two assessments were used to classify all data as either "high" or "low" A/D. Data normality, differences and correlations were tested with the Shapiro-Wilk test, Student's t-test (or the Wilcoxon test), and Spearman test, respectively. The alpha level was set at 0.05 . Results: None of the clinical variables were significantly different when comparing low and high $A / D$ data. In low $A / D$ there was a significant correlation between HADS score and: MFIQ ( $P=0.005, r=0.61)$, and MMO without pain $(P=0.01, r=-0.55)$. Conclusions: Variation in A/D level did not change clinical symptoms or jaw functionality in college students with TMD. Apparently, there is a correlation between TMJ functionality and A/D level, which should be further investigated, taking into account the source of the TMD and including subjects with greater functional limitation.
\end{abstract}

Keywords: Physical therapy specialty. Electromyography. Facial pain. Temporomandibular joint. Anxiety. Depression.

\section{INTRODUCTION}

Temporomandibular disorder (TMD) is a general term that refers to disorders associated with the temporomandibular joint (TMJ) and the masticatory muscles ${ }^{1}$. Due to the complexity of the masticatory system, TMD symptoms may be caused by different physiological and/or psychosocial factors, such as malocclusion and occlusal interferences, alterations in the masticatory muscles, direct trauma to the jaw or TMJ, microtrauma caused by continuous parafunctional habits or alterations secondary to stress $^{1}$

The prevalence of TMD symptoms among the general population is around $40 \%^{15}$. Focusing on university students, Casanova-Rosado, et al. ${ }^{8}$ (2006) found a TMD prevalence of $46.9 \%$ and Shiau and Chang ${ }^{23}$ (1992) reported a prevalence of $42.9 \%$. These results are also applicable to Brazilian students: the reported TMD prevalence for this population ranges from $53.21 \%{ }^{19}$ to $68 \%^{20}$.

TMD is often associated with somatic and 
psychological complaints, including fatigue, sleep disturbances, anxiety, and depression 6,8,24,29. Moreover, psychosocial factors vary according to the etiologic subgroup of diagnosis. In general, these factors are more frequently observed in patients with myogenous TMD $2,21,28$. In 2008, Restrepo, et al. ${ }^{21}$ (2008) reported that children with bruxism had a significantly higher-tension personality and were more prone to anxiety, as well as had more TMD signs and symptoms, than a control group. Bonjardim, et al. ${ }^{6}$ (2005) evaluated TMD patients and found no evidence of either anxiety or depression symptoms according to the Hospital Anxiety and Depression Scale (HADS), although the TMD level was shown to be significantly related to anxiety but not to depression.

The prevalence of mental health problems and subsyndromal symptoms in Australian students ${ }^{25}$ was $19.2 \%$ and $67.4 \%$, respectively. These rates were significantly higher than those of the general population. Psychological distress was associated with disability and lower academic achievement. The identified predictors of distress were full-time status, financial stress, age between and 18 and 34 years-old, being female, and in a subsequent undergraduate year of their degree.

Tsai, et al. ${ }^{27}$ (2002) studied the influence of an experimental stress situation on the electromyographic activity of masticatory muscles. They observed increased masseter activation during stress, which decreased in a relaxing situation. Considering that stress is associated with psychological disturbances such as anxiety and depression ${ }^{13}$ and that there are no published studies observing the modulation of signs and symptoms of TMD according to anxiety and depression level in college students, the objectives of the present study were (1) to verify clinical symptoms and jaw functionality in college students with TMD according to the level of anxiety/depression (A/D) evaluated in two different moments, and (2) to evaluate the correlation between $A / D$ and functionality, maximum mouth opening (MMO), pain and muscle activity.

It was hypothesized that: (1) clinical symptoms would be greater and jaw functionality more impaired in college students with TMD when the level of $A / D$ is higher; (2) this could indicate a possible correlation between $A / D$ and clinical measures of TMD. All subjects were addressed in two different moments in order to obtain data of both high and low A/D level.

\section{MATERIAL AND METHODS}

\section{Study design}

This was a longitudinal study investigating TMD in undergraduate Physical Therapy students from the Federal University of São Carlos (UFSCar), Brazil, according to anxiety and depression level variation during an academic semester.

\section{Subjects}

All of UFSCar's undergraduate Physical Therapy students were invited to participate. A total of 116 volunteers, 32 men and 84 women (mean age 20.87, SD: 1.96 years), signed a term of informed consent and responded to the Fonseca Anamnesis Index (FAI ${ }^{4,12}$. Subjects with moderate or severe signs and symptoms of TMD according to FAI were examined by two trained evaluators, as recommended by the Research Diagnostic Criteria for Temporomandibular Disorders (RDC/TMD) consortium (http://www.rdc-tmdinternational.org). Students not diagnosed with TMD were excluded. Therefore, 19 subjects (18 women and 1 man; mean age of $20.66 \pm 1.59$ years-old) were evaluated at two different moments of the academic semester, with an interval of 4 months. Figure 1 shows the study flowchart.

The study was approved by the local Ethics Committee (permission number 234/2010).

Instruments and evaluation procedures

The RDC/TMD ${ }^{11}$ was created to establish a proper criterion for diagnosing subjects with some

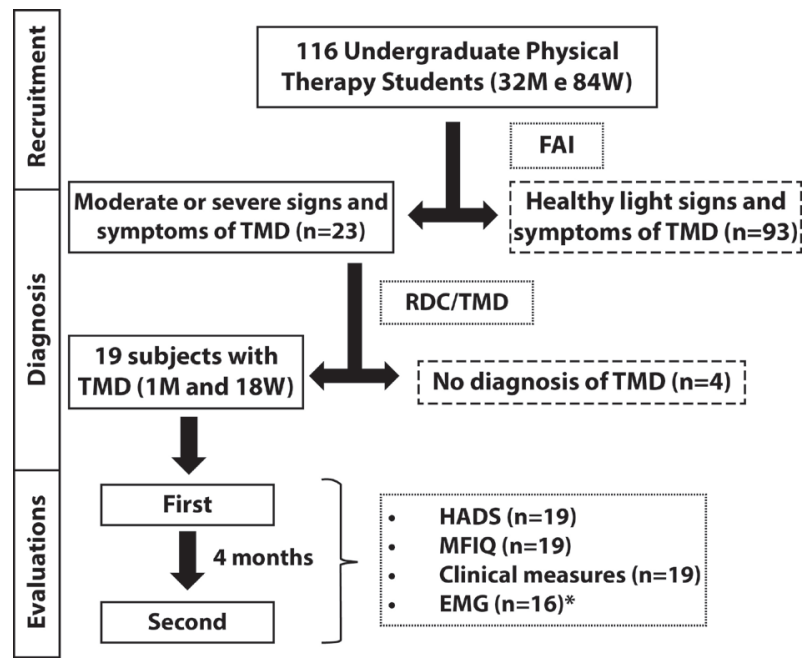

Figure 1- Study flowchart. The descriptions of excluded subjects are in the dashed boxes. Description of the instruments applied at each moment and the number of subjects evaluated are in the dotted boxes

(M=Men, $\quad W=$ Women, $\quad F A l=F o n s e c a \quad$ Anamnesis Index, TMD=Temporomandibular Disorders, RDC/ TMD=ResearchDiagnosticCriteriaforTemporomandibular Disorders, MFIQ=Mandibular Function Impairment Questionnaire, HADS=Hospital Anxiety and Depression Scale, EMG=Electromyography). *Data from 3 subjects were excluded due to technical problems identified during EMG signal analysis 
TMJ compromise. It is a dual-axis system that can be used to classify and quantify both physical and psychosocial components of TMD. The diagnostic system is nonhierarchical and allows for the possibility of multiple diagnoses for a given subject. The three possible types are: muscle disorders (I), disc displacement (II) and joint disorders (III), with types I and III being the most common among TMD patients ${ }^{17}$. This protocol was applied once for diagnostic purposes.

The Hospital Anxiety and Depression Scale (HADS), Mandibular Function Impairment Questionnaire (MFIQ), clinical measurements and electromyography of masticatory muscles were applied/recorded in the two evaluations. The HADS $^{30}$ was translated to Portuguese and validated in 2006'. Each item is scored from 0 to 3 , with a maximum score of 42 points. A high score indicates a high level of $A / D$.

The MFIQ ${ }^{14}$ measures the severity of functional limitation caused by TMD. The score ranges from 0 to 5 - the higher the score, the greater the limitation. The Portuguese version of the questionnaire was validated by Campos, et al. ${ }^{7}$ in 2012.

Some clinical measures included in the RDC/ TMD protocol were also recorded: MMO without pain, MMO and assisted MMO; bilateral palpation of TMJ structures, including masticatory muscles (masseter, temporalis, posterior area of the jaw, submandibular region, lateral pterygoid area and tendon of the temporalis muscle) as well as joint structures (TMJ lateral pole and posterior ligament). A caliper was used for all opening measures. Based on palpation of the TMJ structures, the variable "number of tender points" was calculated.

The electromyography examination (EMG) consisted of recording the electrical activity of the anterior temporal and masseter muscles on the most painful side of each subject's face. For subjects with bilateral involvement, the side to be recorded was randomized. Active single differential surface electrodes (Model \#DE-2.1, Delsys, Boston, MA, USA) with two parallel silver bars (1 $\mathrm{mm} x$ $1 \mathrm{~cm}$ ) separated by $1 \mathrm{~cm}$ were attached to the skin using a double-sided interface (Delsys). The electrode characteristics included: RRMC>80 dB, input impedance $>10^{15} \mathrm{~W}$ in parallel, with $0.2 \mathrm{pF}$, a voltage gain of 10 and noise of $1.2 \mu \mathrm{V}$ (root mean square, RMS). They were placed on the muscle belly parallel to the muscle fibers ${ }^{3}$. On the anterior temporalis muscle, the electrode was fixed vertically along the anterior portion near the coronal suture. On the masseter muscle, the electrode was positioned at the portion with the highest volume, identified by palpation ${ }^{26}$. A square self-adhesive electrode with a $5 \mathrm{~cm}$ side was used as a reference and fixed to the sternal notch. Before attaching the electrodes, the skin was shaved and cleaned with alcohol. The signals were conditioned by the main amplifier (Bagnoli-8 EMG System, Delsys), which provided a gain of 1000 , a bandwidth of 20-450 $\mathrm{Hz}$, and noise of $1.2 \mu \mathrm{V}$ (RMS). The signals were sampled at $2000 \mathrm{~Hz}$ using a PC workstation with a 16-bit A/D card (PCI-6034E, National Instruments Corporation, Austin, TX, USA) and the EMGworks software (version 3.0, Delsys).

Muscle activity was recorded under three conditions: at rest, maximum voluntary isometric contraction (MVIC), and free chewing. Throughout the test, the subjects sat on a chair without head support and were told to keep their hands on their knees and their trunk to the chair back with their head in a natural upright position. For the at-rest condition, the signal was recorded while the subjects maintained their mouths closed without occluding the teeth. Three MVIC trials were then recorded using two rolls of cotton (10 $\mathrm{mm}$ diameter) placed between the upper and lower second premolars/ first molars of the subjects, who were instructed to bite as hard as possible for 5 seconds. Two minutes of rest were allowed between trials. For the chewing condition, the subjects were instructed to chew a piece of parafilm ${ }^{5} 6$ times in order to reduce the volume and to adjust the material. The muscle activity was recorded over a period of 20 seconds, and normalization was based on the MVIC recording. The baseline signal, recorded during rest, was subtracted from the signal recorded during mastication to remove the noise.

\section{Data processing}

The data collected in the HADS and MFIQ instruments, as well as the clinical measures, were computed according to their respective protocols.

The EMG signals were processed using a Matlab ${ }^{\circledR}$ (version 7.0.1, MathWorks Inc., Natick, MA, USA) routine. All signals were band-pass filtered by a fourth-order zero-lag Butterworth filter at 20-400 $\mathrm{Hz}$. They were converted into RMS using $20 \mathrm{~ms}$ windows with $50 \%$ overlap. These data were normalized by the highest RMS value of the three MVIC. The RMS value of the signal obtained at rest was subtracted from the signals in order to remove the noise. The average RMS amplitude of each muscle and the activation time were also calculated (total time of muscle activity during chewing).

Data obtained from each subject in the two evaluations were divided into two data sets, according to HADS score: low A/D and high A/D. The HADS scores were classified by direct comparison of individual data. After that, all other variables were also divided in low and high A/D.

\section{Statistical analysis}

Data analysis was performed with Statistica software (v7, StatSoft Inc., Tulsa, OK, USA). The 
Shapiro-Wilk test was used to verify the normality of the data. In order to compare data from the same subject at two different moments (high A/D and low $A / D)$, either Student's $t$-test for paired samples or the Wilcoxon test was applied, according to data distribution. The effect size was also calculated, and classified as small for values between 0.20 and 0.49 ; moderate for values between 0.50 and 0.79 , and large for values larger than $0.8^{10}$. For both data sets (high $A / D$ and low $A / D$ ), the correlation between all variables and the HADS score was tested using Spearman correlation coefficients (SC). The strength of the relationship was considered "weak" when SC ranged from 0 to 0.25 , "low" from 0.26 to 0.49 , "moderate" from 0.50 to 0.69 , "high" from 0.70 to 0.89 and "very high" above $0.90^{22}$. The alpha level was set at 0.05 for all analyses.

\section{RESULTS}

The FAI results indicated a $70.6 \%$ prevalence of TMD signs and symptoms (from light to severe) among the evaluated students. Most ( $n=60$; $51.6 \%$ ) were classified as having light signs and symptoms of TMD. Fifteen (13.0\%) were classified as having moderate and eight (7\%) as having severe signs and symptoms of TMD. The other 33 students $(28.4 \%)$ were classified as healthy.

According to the RDC/TMD (Axis I and Axis II), five subjects presented TMD type I (muscle disorders), five TMD type III (disc displacements), and nine mixed TMD. No subject presented only disc displacements (type II). Six subjects presented unilateral compromise on the right side, seven on the left side and six presented bilateral compromise.

The data from the questionnaires, clinical measurements and EMG for both high and and low A/D are shown in Table 1.

There was a significant difference between the HADS score for low and high A/D (T test, $P=0.0005$, $\mathrm{ES}=0.83$ ), with a mean difference of $40 \%$. The MFIQ score was higher for the high A/D, but there was no significant difference between the low and high $A / D$ conditions ( $T$ test, $p=0.08, E S=0.582$ ). The same was observed for the MMO measures (Table 1).

EMG data are shown in Table 2. Since some signals were compromised by the presence of electrical noise, three subjects were excluded.

Table 1- Mean, standard deviation (SD), 95\% confidence interval (CI), p-value and effect size of HADS and MFIQ scores, $\mathrm{MMO}$ in millimeters and number of tender points $(n=19)$

\begin{tabular}{ccccccc}
\hline Variables & \multicolumn{2}{c}{ High A/D } & \multicolumn{2}{c}{ Low A/D } & P value & Effect size \\
& mean (SD) & $\mathbf{9 5 \%}$ Cl & mean (SD) & $\mathbf{9 5 \% ~ C l ~}$ & \\
\hline HADS score & $12.10(6.91)$ & $8.7-15.4$ & $8.57(6.25)$ & $5.55-11.58$ & 0.0005 & 0.838 \\
\hline MFIQ score & $1.21(1.18)$ & $0.6-1.71$ & $0.79(1.08)$ & $0.26-1.31$ & 0.08 & 0.582 \\
$\begin{array}{c}\text { MMO without } \\
\text { pain }\end{array}$ & $31.15(9.38)$ & $26.6-35.6$ & $30.21(11.00)$ & $24.9-35.5$ & 0.75 & 0.141 \\
MMO & $42.05(6.36)$ & $38.9-45.1$ & $39.73(8.25)$ & $35.7-43.7$ & 0.19 & 0.467 \\
$\begin{array}{c}\text { MMO with } \\
\text { assistance }\end{array}$ & $45.26(6.51)$ & $42.1-48.3$ & $43(7.61)$ & $39.3-46.6$ & 0.23 & 0.492 \\
$\begin{array}{c}\text { No of tender } \\
\text { points }\end{array}$ & $12(5.00)$ & $9.5-14.4$ & $12.73(6.59)$ & $12.7-15.9$ & 0.47 & -0.184 \\
\hline
\end{tabular}

MFIQ=Mandibular Function Impairment Questionnaire; HADS=Hospital Anxiety and Depression Scale, MMO=maximum mouth opening

Table 2- Mean, standard deviation (SD), 95\% confidence interval (CI), p-value and effect size of EMG data obtained during free chewing, recorded on the affected side $(n=16)$

\begin{tabular}{cccccccc}
\hline \multicolumn{1}{c}{ Variables } & \multicolumn{2}{c}{ High A/D } & \multicolumn{2}{c}{ Low A/D } & P-value Effect Size \\
\hline Mean RMS (\%MVIC) & AT & $0.33(0.17)$ & $0.23-0.42$ & $0.31(0.13)$ & $0.24-0.37$ & 0.8 & 0.195 \\
& M & $0.27(0.16)$ & $0.18-0.35$ & $0.25(0.13)$ & $0.18-0.31$ & 0.8 & 0.208 \\
Activation time (s) & AT & $13.03(4.82)$ & $10.4-15.5$ & $12.54(5.01)$ & $9.87-15.2$ & 0.77 & 0.157 \\
& M & $14.09(4.42)$ & $11.7-16.4$ & $15.99(5.32)$ & $13.1-18.8$ & 0.14 & -0.59 \\
\hline
\end{tabular}

$\mathrm{AT}=$ anterior temporalis; $\mathrm{M}=$ masseter; $\mathrm{MVIC}=$ maximum voluntary isometric contraction; $\mathrm{A} / \mathrm{D}=$ anxiety/depression EMG=Electromyography 


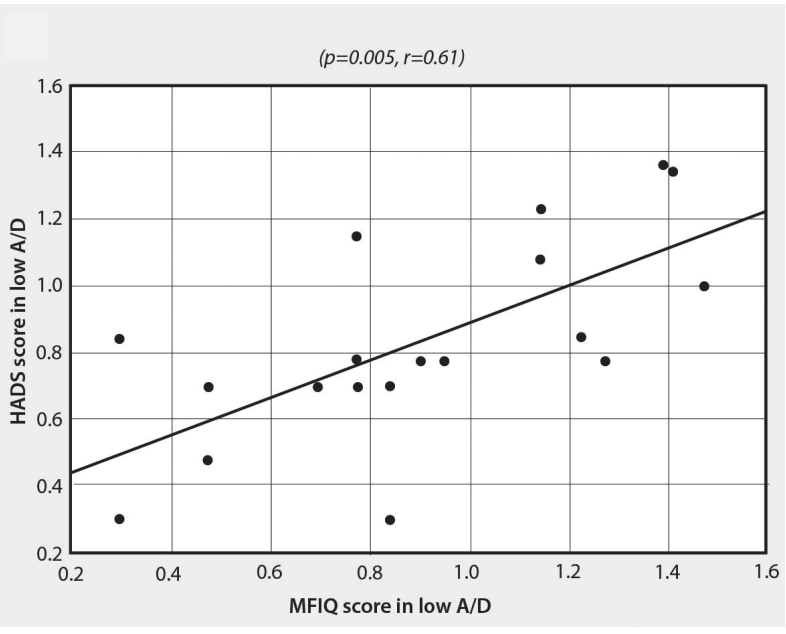

Figure 2- Correlation analysis of HADS score and MFIQ in low $A / D$

HADS=Hospital Anxiety and Depression Scale, $M F I Q=M a n d i b u l a r$ Function Impairment Questionnaire, $\mathrm{A} / \mathrm{D}=$ Anxiety/Depression

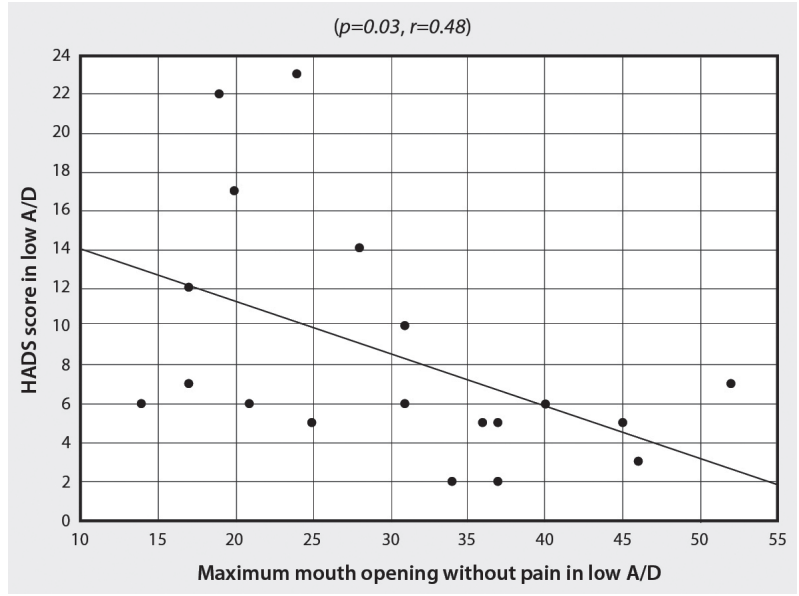

Figure 3- Correlation analysis of HADS score and MMO without pain in low $A / D$

HADS=Hospital Anxiety and Depression Scale, $\mathrm{MMO}=$ maximum mouth opening, $\mathrm{A} / \mathrm{D}=$ Anxiety $/$ Depression

Table 3- Spearman correlation coefficient and p-values between HADS score in high and low A/D for all variables addressed

\begin{tabular}{ccccc}
\hline & & High A/D & & Low A/D \\
& $\mathbf{r}$ & p-value & $\mathbf{r}$ & p-value \\
\hline MFIQ score & 0.21 & 0.38 & 0.61 & 0.005 \\
MMO without pain (mm) & -0.13 & 0.58 & -0.55 & 0.01 \\
MMO (mm) & -0.07 & 0.75 & -0.45 & 0.053 \\
MMO with assistance (mm) & -0.14 & 0.54 & -0.44 & 0.053 \\
No of tender points & 0.07 & 0.76 & 0.24 & 0.3 \\
Mean RMS - AT (\%MVIC) & 0.2 & 0.44 & 0.21 & 0.41 \\
Mean RMS - M (\%MVIC) & -0.04 & 0.87 & 0.23 & 0.37 \\
\hline Activation time - AT (s) & -0.14 & 0.58 & -0.01 & 0.95 \\
\hline Activation time - M (s) & -0.33 & 0.2 & -0.01 & 0.95 \\
\hline
\end{tabular}

AT=anterior temporalis; $\mathrm{M}=$ masseter; $\mathrm{MMO}=$ maximum mouth opening; $\mathrm{MFIQ=Mandibular}$ Function Impairment Questionnaire; HADS=Hospital Anxiety and Depression Scale, A/D=Anxiety/Depression

Thus, the data from 16 subjects were used in this analysis. No difference was found in EMG data when comparing high and low A/D ( $P>0.05)$.

There was no correlation between the variables in high A/D. Otherwise, as presented in Table 3, the correlation between HADS and MFIQ scores for low A/D condition was positive and moderately significant ( $S C, P=0.005, r=0.61$ ) - Figure 2. In low $A / D$, a negative and moderately significant correlation was also observed between HADS score and MMO without pain (SC, $\mathrm{P}=0.01, \mathrm{r}=-$ 0.55) - Figure 3. Moreover, two variables presented borderline $\mathrm{p}$-values: $\mathrm{MMO}$ and number of tender points (Table 3 ).

\section{DISCUSSION}

The results of this study showed that even though college students with TMD presented A/D changes during the semester according to the HADS, clinical variables directly related to TMD did not change during the same period. This result refutes the first hypothesis of the study. Furthermore, in the low A/D condition, a significant correlation was found between the level of $A / D$ and some clinical measurements (MFIQ and MMO without pain), which partially confirms the second hypothesis.

The significant variation of anxiety and depression levels identified between the two evaluations was expected, which may reflect emotional oscillations 
due to personal and academic activities that affected anxiety and depression levels during the semester. However, based on the available literature, we expected that the clinical variables related to TMD would also change. A coexistence of signs and symptoms of TMD and psychological complaints is reported in the literature, and some studies dealing with this subject have already been briefly described above $6,8,24,29$. Moreover, Monteiro, et al. ${ }^{18}$ (2011) also identified a significant correlation between anxiety levels and degree of orofacial pain in university students, although they used different instruments to classify and quantify the observed variables. Giannakopoulos, et al. ${ }^{14}$ (2010) used HADS to investigate the prevalence of anxiety and depression in subjects with TMD type I (muscle disorders), TMD type III (joint disorders) and healthy controls, as well as the prevalence of chronic facial pain. Although the anxiety scores of both TMD groups were similar, there was a higher prevalence of depression in those with TMD type I than with type III, particularly among women. Finally, Bonjardim, et al. ${ }^{6}$ (2005) evaluated TMD patients and found no evidence of either anxiety or depression among the subjects in their sample. However, they found that TMD level was significantly related to anxiety.

Bearing in mind the positive relationship between TMD and psychological factors, higher levels of anxiety and depression should lead to a lower MMO, reduced functionality and a greater number of tender points, as well as greater electrical activity in the masticatory muscles ${ }^{27}$. This, however, did not turn out to be the case in our sample, which could be explained by the HADS scores of subjects in the high A/D condition. According to the literature, HADS scores over 13 points indicate the presence of anxiety and depression ${ }^{16}$. The high A/D reported in this study refers to a mean HADS score of 12.10: seven of the 19 subjects scored over 13 . So, even though the subjects in the high A/D condition had elevated HADS scores, most of them did not present anxiety or depression according to HADS. In most of the subjects, the variation observed between "high" and "low" A/D occurred in a "healthy" condition. Therefore, the variation of $A / D$ was probably not enough to change clinical TMD measures (MMO, functionality, number of tender points and muscle activation).

Considering the association between stress and psychological disturbances such as anxiety and depression ${ }^{13}$, it was expected a variation of the masseter and temporal electrical activity according to the level of A/D. However, this behavior was not observed, probably due to the small variation in $A / D$. It is likely that the variation observed among the subjects was not enough to change muscular activation. In addition to that, we believe that the instrument applied to measure anxiety and depression does not evaluate the same psychological dimension as other studies that have investigated the association between EMG and stress. Future studies intending to explore the variation of psychological factors and their influence on clinical TMD variables should use other questionnaires than the HADS or create stressful situations, just as studies reported in the literature ${ }^{27}$.

Even though difference in clinical TMD measures at different $A / D$ levels was not identified, the data did indicate a correlation between A/D level and some variables, particularly in the low $A / D$. A moderate negative correlation between MMO without pain and HADS score was observed, as well as a moderate positive correlation between TMJ functionality (MFIQ score) and HADS score. The fact that the correlations were observed only in those with low $A / D$ may be related to the heterogeneity of the sample. Considering that women with clinical symptoms of TMD type I have higher rates of depression ${ }^{14}$, the correlation between $A / D$ and TMD clinical variables would be better investigated if taken into account the type of TMD. The relatively small number of subjects did not allow the sample to be divided according to TMD type. Furthermore, it should be pointed out that even though all of the subjects were diagnosed with TMD, most of them did not present relevant functional impairment of the TMJ. We believe that the investigation of subjects with greater functional limitation could provide additional and more conclusive information.

Finally, the study has certain limitations that should be considered. The first is the size and the heterogeneity of the sample. A larger sample would probably bring up more conclusive results regarding TMJ functionality (evaluated by the MFIQ). Moreover, further studies should consider the type of TMD (according to RDC/TMD) in order to assess the influence of anxiety and depression on clinical indicators of dysfunction. The evaluation of subjects without TMD would also provide additional information and help to interpret the results. Another limitation is the fact that the subjects were not severely anxious/depressed. It is possible that the variation in psychosocial indicators was not enough to influence the clinical TMD variables analyzed in the study. The subjects were recruited from a sample of students who were not under treatment for orofacial pain and had not been referred for rehabilitation by a doctor or a dentist. Therefore, further studies should evaluate subjects with higher levels of anxiety and depression and functional impairment. 


\section{CONCLUSIONS}

Although the college students' anxiety and depression level changed over the 4 month academic semester, no difference in TMD signs and symptoms was observed in subjects with either high or low levels of anxiety and depression. The correlation identified between anxiety and depression and some clinical variables in the low A/D condition suggests an association between these factors, which should be better investigated, taking the origin of TMD into consideration, in subjects with greater functional impairment.

\section{ACKNOWLEDGMENTS}

The authors are grateful to Professor Débora Bevilaqua Grossi for a careful reading of the research project and for suggestions, and to São Paulo Research Foundation (FAPESP) for financial support (grant 2010/15872-5).

\section{REFERENCES}

1- American Society of Temporomandibular Joint Surgeons (ASTJS). Guidelines for diagnosis and management of disorders involving the temporomandibular joint and related musculoskeletal structures. Cranio. 2003;21(1):68-76

2- Auerbach SM, Laskin DM, Frantsve LM, Orr T. Depression, pain, exposure to stressful life events, and long-term outcomes in temporomandibular disorder patients. J Oral Maxillofac Surg. 2001; 59:628-33.

3- Bevilaqua-Grossi D, Chaves TC, Lima-Duarte K, Oliveira AS Atividade eletromiográfica dos músculos masseter e temporal anterior de crianças com mordida cruzada posterior unilateral (MCPu). Rev Bras Fisioter. 2005;9(3):257-63.

4- Bevilaqua-Grossi D, Chaves TC, Oliveira AS, Monteiro-Pedro V. Anamnestic index severity and signs and symptoms of TMD. Cranio. 2006;24(2):112-8.

5- Biasotto-Gonzalez DA, Berzin F, Costa KM, Gonzalez TO. Electromyographic study of stomatognathic system muscles during chewing of different materials. Electromyogr Clin Neurophysiol. 2010;50(2):121-7

6- Bonjardim LR, Gavião MB, Pereira LJ, Castelo PM. Anxiety and depression in adolescents and their relationship with signs and symptoms of temporomandibular disorders. Int J Prosthodont. 2005; 18:347-52.

7- Campos JA, Carrascosa AC, Maroco J. Validity and reliability of the Portuguese version of Mandibular Function Impairment Questionnaire. J Oral Rehabil. 2012;39;377-83.

8- Casanova-Rosado JF, Medina-Solís CE, Vallejos-Sánchez AA, Casanova-Rosado AJ, Hernández-Prado B, Ávila-Burgos L. Prevalence and associated factors for temporomandibular disorders in a group of Mexican adolescents and youth adults. Clin Oral Investig. 2006;10:42-9.

9- Castro MM, Quarantini L, Batista-Neves S, Kraychete DC, Daltro C. Validity of the hospital anxiety and depression scale in patients with chronic pain. Rev Bras Anestesiol. 2006;56:470-7.

10- Cohen J. Statistical power analysis for behavioural sciences. $2^{\text {nd }}$ ed. Hillsdale: Lawrence Eribaum Associates; 1988.

11- Dworkin SF, LeResche L. Research diagnostic criteria for temporomandibular disorders: review, criteria, examinations and specifications, critique. J Craniomandib Disord. 1992;6:301-55.
12- Fonseca DM, Bonfante G, Valle AL, Freitas SF. Diagnóstico pela anamnese da disfunção craniomandibular. RGO (Porto Alegre). 1994;42:23-8.

13- Gameiro GH, Silva Andrade A, Nouer DF, Ferraz de Arruda Veiga MC. How may stressful experiences contribute to the development of temporomandibular disorders? Clin Oral Investig. 2006;10:261-8.

14- Giannakopoulos NN, Keller L, Rammelsberg P, Kronmüller $\mathrm{KT}$, Schmitter M. Anxiety and depression in patients with chronic temporomandibular pain and in controls. J Dent. 2010;38:369-76. 15- Gonçalves DA, Dal Fabbro AL, Campos JA, Bigal ME, Speciali JG. Symptoms of temporomandibular disorders in the population: an epidemiological study. J Orofac Pain. 2010;24:270-8.

16- Hinz A, Brähler E. Normative values for the hospital anxiety and depression scale (HADS) in the general German population. ] Psychosom Res. 2011;71:74-8.

17- Manfredini D, Arveda N, Guarda-Nardini L, Segù M, Collesano V. Distribution of diagnoses in a population of patients with temporomandibular disorders. Oral Surg Oral Med Oral Pathol Oral Radiol. 2012;114:e35-41.

18- Monteiro DR, Zuim PR, Pesqueira AA, Ribeiro PP, Garcia $A R$. Relationship between anxiety and chronic orofacial pain of temporomandibular disorder in a group of university students. J Prosthodont Res. 2011;55(3):154-8.

19- Nomura K, Semprini M, Siéssere S, Eduardo J, Hallak C, Cecilio $S$, et al. Use of the Fonseca's questionnaire to assess the prevalence and severity of temporomandibular disorders in Brazilian dental undergraduates. Braz Dent J. 2007;18:163-7. 20- Pedroni CR, Oliveira AS, Guaratini MI. Prevalence study of signs and symptoms of temporomandibular disorders in university students. J Oral Rehabil. 2003;30(3):283-9.

21- Restrepo CC, Vásquez LM, Alvarez M, Valencia I. Personality traits and temporomandibular disorders in a group of children with bruxing behaviour. J Oral Rehabil. 2008;35:585-93.

22- Russel E, Carter RE, Lubinsky J, Domholdt E. Rehabilitation research: principles and applications. St. Louis: Elsevier Saunders; 2005.

23- Shiau YY, Chang C. An epidemiological study of temporomandibular disorders in university students of Taiwan. Community Dent Oral Epidemiol. 1992;20:43-7.

24- Sipilä K, Mäki P, Laajala A, Taanila A, Joukamaa M, Veijola J. Association of depressiveness with chronic facial pain: a longitudinal study. Acta Odontol Scand. 2012;71:644-9.

25- Stallman HM. Psychological distress in university students: a comparison with general population data. Aust Psychol. 2010;45(4):249-57.

26- Tartaglia GM, Silva MA, Bottini S, Sforza C, Ferrario VF. Masticatory muscle activity during maximum voluntary clench in different research diagnostic criteria for temporomandibular disorders (RDC/TMD) groups. Man Ther. 2008;13:434-40.

27- Tsai CM, Chou SL, Gale EN, McCall WD Jr. Human masticatory muscle activity and jaw position under experimental stress. J Oral Rehabil. 2002;29:44-51.

28- Visscher CM, Lobezzoo F, de Boer W, van der Meulen M, Naeije $M$. Psychological distress in chronic craniomandibular and cervical spinal pain pacients. Eur J Oral Sci. 2001;209:165-71.

29- Yap AU, Dworkin SF, Chua EK, List T, Tan KB, Tan HH. Prevalence of temporomandibular disorder subtypes, psychologic distress, and psychosocial dysfunction in Asian patients. J Orofac Pain. 2003; 17:21-8.

30- Zigmond AS, Snaith RP. The hospital anxiety and depression scale. Acta Psychiatr Scand. 1983;67:361-70. 